\title{
La punizione esemplare dello studente Formiggini nel 1896
}

Un documento d'archivio del liceo Galvani di Bologna, coperto da riservatezza, riletto dopo più di un secolo, permette di rivelare le dinamiche che stanno dietro una punizione esemplare inflitta ad uno studente che ha scritto una parodia dell'Inferno dantesco. Il protagonista è il giovane Angelo Fortunato Formiggini, ebreo modenese, editore in Roma da "grande", suicida nel 1938 per protesta contro le leggi razziali.

A confidential document from the archive of the Liceo Galvani in Bologna, rediscovered after more than a century, reveals the dynamics behind an exemplary punishment inflicted on a student who wrote a parody of Dante's Inferno. The student was the young Angelo Fortunato Formiggini, Jewesh from Modena and publisher in Rome as an adult, who commetted suicide in 1938 to protest against racial laws.

La vicenda si consuma tra il novembre e il dicembre 1896 nelle severe aule del Regio liceo Galvani di Bologna dove un brillante studente dell'ultimo anno ha composto una parodia dell'Inferno dantesco, in cui ha incluso per burla tutti i compagni, i professori, il bidello e il preside e ne ha distribuito copie con dedica a tutti, orgoglioso del proprio prodotto. Il preside vuole una punizione esemplare. Lo studente si sente ingiustamente vittima di un complotto clericale ma i documenti d'archivio offrono tutt'altra ricostruzione, rivelando una dinamica dei fatti diversa da quella creduta dai giovani e dai lettori dei giornali.

Il protagonista è il diciottenne Angelo Fortunato Formiggini, studente modenese di ricca famiglia ebraica, trasferito a Bologna fin dal 1895 per concludervi gli studi liceali e compiervi quelli universitari. Coprotagonisti sono un energico preside, Emilio Roncaglia, anch'egli modenese, di famiglia liberale, prestato alla scuola 
dopo una laurea in giurisprudenza per le sue opere letterarie, e sei professori autorevoli: il giovane Angelo Solerti (italiano), famoso per i suoi studi su Tasso e sul melodramma, il cattolico Carlo Tincani (lettere classiche), temutissimo per severità e rigore, il conte senese Giuseppe Martinozzi (storia civile), sostenitore di valori laici di solidarietà e umanismo, Luigi Lavi Salvetti (filosofia), Riccardo Boselli (matematica) e infine il cattolicissimo Giuseppe Casati (fisica e storia naturale). Lo studente Formiggini ha frequentato la seconda liceo con ineccepibile condotta e ottimo profitto. E' un ragazzo esuberante, cordiale, con molti amici, incline a sorridere di tutto con simpatia. Si potrebbe sostenere che è in potenza l'uomo che sarà, cioè il sostenitore laico della benevolenza universale, della fratellanza tra gli uomini e tra i popoli, l'estimatore del riso come ingrediente fondamentale del buon vivere. All'inizio della terza liceo decide di divertire e divertirsi con la sua parodia dantesca, che intitola La Divina Farsa. Letto con l'odierna sensibilità il poemetto è gustosissimo, se non fosse per certe allusioni a vizi dei compagni. Del resto all'inferno non si può collocare gente virtuosa.

\section{II corpo del reato}

La Divina Farsa ovvero La Descensione ad Inferos 1 , di Formaggino da Modena è proposta come opera inedita di secolo incerto, fonte d'ispirazione dello stesso Dante, e racconta in tredici canti lo "smarrimento" dello studente Formiggini, che ha al suo fianco, nei panni di Virgilio, l'amico Balducci, inviato dal cielo da una Beatrice che è la più bella e la più brava delle compagne di classe, Emma Honig, ungherese di nascita, poi laureata con lode in lettere con Carducci. Varcato il gran portone del Galvani si entra nei luoghi infernali ove «per trastullo ciascun professore Si mette a dar dei quattro ed a bocciare» (Canto II). Nel quadro successivo degli ignavi mi pare innocua l'invettiva contro i borghesi ma assai imprudente l'affondo anticlericale del «gran fetore d'incenso e di libri da messa» (Canto III). Decisamente divertente il bidello-portinaio trasformato in Caronte e gli studenti "incompresi" dai professori al posto degli spiriti magni. Il Canto V è interamente occupato da professori. E se il professore di filosofia è risolto in pochi versi, il professore di storia, nel ruolo di Minosse - «Sta Martinoz eternamente e boccia» - occupa il resto del canto ed è ritratto con viva simpatia. «Quel gran conoscitor delle peccata», con gentilezza sbarra il cammino ma, costretto a cedere alla volontà del preside, «ch'ha lo stile di far ciò che gli piace e che gli pare», si limita 
a ricordare di avere con sé il quaderno degli appunti. L'estro bizzarro di Formaggino sfiora uno dei punti più alti nel secondo girone dei lussuriosi (Canto VI), immaginati come ciclisti lanciati in folle corsa su bici senza freni "Prinetti", un gioiello tecnologico di fine secolo. Su un tandem impazzito, nei panni di Paolo e Francesca, stanno Ghedini e Dellamore, che insieme spesso facevano fughino. Ma le allusioni ai "peccatucci" dei lussuriosi non devono essere sfuggite alla «Santa Inquisizione» di preside e professori. Non sono le due teste del professore Boselli (Algebra e Geometria), ad essere dispiaciute o le esortazioni «eterne» di Casati alla moderazione, divenute la vera punizione dell'iracondo Cazzola, nudo sul ghiaccio, o le ali Solerti di quell'Angelo che sferza il posteriore di Crispolti. E neppure il Canto XIII, in cui Formaggino trova l'occasione di far rivivere un episodio del 23 dicembre 1895, un "fughino" della classe per evitare la lezione di greco pomeridiana - il pomeriggio prima delle vacanze di Natale. I pochi che si sono presentati, $i$ «traditori degli amici», vengono sbranati da Lucifero-Tincani. Esagerata risulta l'insistenza sull'ubriachezza di Longhini (Canto VII) ma soprattutto è offensiva la rappresentazione del «sodomita clericale» Crispolti (Canto XII). Non c'è simpatia neppure per il "superbo" Corsi (Canto VIII). Simpatia c'è, invece, per Ballini-Pier delle Vigne, collezionista di «codici rari» e di «libracci antichi», nonché di lenti per decifrarli. Suicida il Ballini perché due sorci «fecer gustosissimo banchetto del suo tesoro». Ambrogio Ballini, a cui è dedicato il canto XI, laureato in lettere a Bologna, diventerà professore di sanscrito e resterà in affettuosa corrispondenza per anni con Formiggini. Il poeta Formaggino ha pure usato due parolacce (Canto II, v. 14 - Canto VIII, v. 3), entrambe con significato metaforico, per alludere l'una alla fortuna o buona sorte e l'altra agli sfortunati un poco ingenui, con particolare riguardo all'autore. Che dire? L'uso, dopo un secolo, le ha consegnate all'uso quotidiano. Ma nel 1896... Del tutto tranquillo non deve essere l'autore se dall'amico Longhini si lascia fare una predizione inquietante: «T'avviso che li Spiriti sovrumani Stannoti ordendo una brutta sorpresa, S'or non intendi, intenderai domani». Mai predizione fu più vera.

\section{La punizione esemplare nei documenti d'archivio}

L'effetto prodotto dalla distribuzione capillare della Divina farsa convince il preside Roncaglia, guida morale del liceo, che è necessario intervenire con un provvedimento disciplinare. Convoca il consiglio dei professori in "adunanza stra- 
ordinaria". Il verbale, in data 2 dicembre $1896,{ }^{2}$ ore 5 pomeridiane, redatto dal professor Solerti, registra che "Il Consiglio conviene che l'opuscolo "La divina farsa" può essere gravemente offensivo per taluno degli alunni nominati.» $\mathrm{Ne}$ deduciamo che i professori escludono che ci siano riferimenti ingiuriosi a loro, ma consentono col preside che alcuni condiscepoli del Formiggini e loro famiglie possano sentirsi offesi. Stante l'ora tarda il Preside annunzia che sospenderà domattina il giovane per cinque giorni e riconvocherà il Consiglio. L'alunno in presidenza si mostra pentito e disposto a far ritorno a Modena ma dentro di sé comincia a pensare ad una "congiura cattolica", idea che non lo abbandonerà più. Due giorni dopo, Roncaglia riunisce il consiglio 3 . È il 4 dicembre. La discussione si preannuncia tesissima al punto che il professor Martinozzi subito «si assenta per malessere». E vale la pena di notarlo questo particolare per quanto si osserverà in seguito. Il Preside riferisce di avere sospeso provvisoriamente l'alunno Formiggini, e che il medesimo ha dichiarato di ritirarsi. Ma la gravità del reato merita un castigo adeguato per tutelare il buon nome del liceo. Perciò propone un provvedimento di espulsione, che mette in discussione e ai voti, pensando ad un esito scontato. Così non è.

[...] mentre i suddetti insegnanti si dichiarano fermi nel volere che lo studente Formiggini non rientri assolutamente, né oggi né più tardi, in questo Liceo, non credono di poter votare in favore della proposta del signor Preside per le seguenti considerazioni:

$-1^{\wedge}$ Perché la presentazione di un esemplare dell'opuscolo fatta dal Formiggini agli insegnanti con dedica rispettosa, riverente e riconoscente, prova che egli era per lo meno in grande parte incosciente della mancanza commessa.-2^ Perché l'ottimo profitto e la condotta corretta dell'alunno nell'anno prossimo passato attenuano di molto la odierna mancanza.- $3^{\wedge}$ Perché l'allontanamento dal Liceo Galvani, per il Formiggini che avrebbe desiderato terminare gli studi in questa città e perciò venuto espressamente da Modena sua patria e residenza, basterà ad assicurare da parte sua una condotta corretta nei pochi mesi che gli mancano per terminare il corso liceale. $-4^{\wedge}$ Perché in vari modi il giovane Formiggini ha mostrato di essere pentito del fallo commesso e di valutarne, per quanto troppo tardi, l'importanza e la gravità. Per questa ragione i sopraindicati professori pregano il Preside di ritirare la sua proposta, ritenendo che inflitta una punizione grave e allontanato l'alunno dal Liceo si sia pienamente raggiunto il fine di provvedere al buon andamento dell'istituto.

La difesa lucida ed articolata dello studente porta alla bocciatura della proposta del preside e avvia il secondo tempo della discussone, non meno insidiosa, sulla pena da infliggere al colpevole al posto di quella estrema, appena sventata. 
Il prof. Tincani aggiunge altre considerazioni [...] in favore dell'alunno e con ciò spiega il suo voto contrario alla proposta del Preside, parendogli che sia sufficente (sic) che l'alunno muti liceo dopo sospeso per un numero di giorni da determinarsi.

La proposta di Tincani si rivela decisiva per salvare la conclusione degli studi di Formiggini e apre la strada ai colleghi.

[...] il prof. Casati propone allora [...] la sospensione temporanea, ritenendo che con l'uscita del Formiggini dall'istituto sia raggiunto lo scopo di metterlo in condizione di poter tornare a quella correttezza di condotta, dalla quale più per leggerezza che per altro si è allontanato.

Il prof. Solerti, quasi per costringere il giovane a questa buona condotta nella scuola dove si recherà, completa la proposta indicando la sospensione per giorni 40, che è il massimo concesso dalla legge, perché superandosi quel numero di giorni l'alunno resta senz'altro escluso dalla prima sessione d'esame.

Dando il massimo della pena minore Formiggini potrà presentarsi all'esame di licenza liceale nella sessione estiva, poiché la sua sospensione cade, in larga misura, entro le vacanze natalizie e il nullaosta può essere dato nella prima metà di gennaio. Col contributo decisivo dei cattolici (Tincani e Casati) ha vinto la linea moderata e perbenista che evita il danno massimo voluto dall'intransigenza di Roncaglia ma non vuole giustificare il gioco divertito e certamente imprudente del Formiggini.

\section{Le reazioni degli studenti e la narrazione dei giornali}

L'episodio diviene di dominio pubblico fin dal 2 dicembre - che è il giorno in cui lo studente è convocato in presidenza. Formiggini si convince di essere stato vittima dell'ala cattolica del corpo insegnante e soprattutto del moralista Casati e di essere stato strenuamente difeso da Martinozzi, il professore da lui più amato, e trasmette questa percezione ai suoi compagni. Gli studenti, liceali e universitari, mettono a rumore la città. Formiggini viene invitato a leggere pubblicamente la Divina farsa in un'aula dell'università, in mezzo a vere ovazioni. Si organizza un corteo di circa 200 studenti che inscenano una dimostrazione e, sulla base delle voci che si sono diffuse, si dirigono vero la casa del prof. Casati, in via Mazzini 22, per fischiarlo, ritenendolo il responsabile della punizione insieme al preside Roncaglia. E qui sta il primo errore. Poi il corteo, che a torto riconosce come unico difensore Martinozzi, di fatto assente alla discussione, si dirige verso via de' Poeti 2, ove egli abita, per applaudirlo sotto casa e infine sotto il "Galvani" fa partire gli ultimi fischi verso la presidenza. Il tutto si conclude, la sera, con una bicchierata alla fiaschetteria Semprini, moderata nei toni dallo stesso Formiggini che, all'indomani, parte per Modena. Risulta chiaro che Formiggini, gli studenti e 
i cittadini bolognesi, informati dai giornali, hanno una percezione falsificata della dinamica degli eventi e dell'orchestrazione della punizione. Tanto "Il Resto del Carlino" quanto la "Gazzetta dell'Emilia" e "Avvenire" riportano la notizia delle dimostrazioni studentesche in data 6 dicembre 1896, accogliendo la narrazione degli studenti. Nei giorni successivi ne danno informazione anche "Il Panaro" e "Il Risveglio" (organo della Federazione socialista romagnola) e persino il "Cristoforo Colombo", quotidiano italiano di New York. "Bologna che ride" dà la notizia del provvedimento concludendo che «se [Formiggini] si fosse mostrato una testa di c...avolo gli avrebbero dato la medaglia».

\section{Due righe conclusive}

La ricerca, condotta in un archivio scolastico insieme agli alunni, ha un elemento psicologico di forza nella istintiva immedesimazione dello studente col protagonista, creativo, imprudente e alla fine vittima, come si sentono spesso gli adolescenti. Il suo vero interesse, però, sta nel rendere possibile il confronto tra lo svolgersi degli eventi nella riservatezza del consiglio dei professori e la loro percezione all'esterno, che alimenta una narrazione falsa degli stessi ma ampiamente diffusa ${ }^{4}$. Oggi, più che mai, avremmo bisogno di documenti d'archivio che in presenza di narrazioni mediatiche aggressive, rancorose, mistificanti potessero ricondurci alla ricostruzione fondata delle vicende, azzerando dietrologie, fraintendimenti, distorsioni e falsificazioni anche in buona fede.

\section{Bibliografia}

Berti Arnoaldi Veli U. 2012, Morire per ridere, in I quaderni di cultura del Galvani 2011/2012, 2, 89-95

Formiggini A. F. 2012, La Divina Farsa, in I quaderni di cultura del Galvani 2011/2012, $2,31-53$

Gaspari M. 2012, “40 piccoli giornetti di sospensione”. La punizione esemplare dello studente Formiggini al Liceo Galvani, in I quaderni di cultura del Galvani 2011/2012, 2, 57-89 